\title{
Improving patient follow-up after inpatient stay
}

\author{
Katie Wimble - Ashford and St Peters NHS Foundation Trust, Keefai Yeong
}

\begin{abstract}
Follow-up is a vital part of ongoing patient safety. It allows for subsequent investigations to be checked and acted upon, encourages specialist review of patients and ensures that patients with chronic conditions receive the appropriate secondary care input. This study aims to highlight and quantify current problems with how follow-up arrangements are made within our hospital and provide a suitable solution to ensure that these problems are minimised. 20 sets of clinical notes were analysed for plans of follow-up and then compared with the discharge summaries produced. Hospital computer systems were used to find out which interventions happened, and when, to get the baseline data. A simple followup prompt sheet was introduced and a further 20 sets of notes were audited to complete the study. Patient follow-up improved after the introduction of a simple follow-up prompt sheet but highlighted the need for a complete change in the way follow up is arranged at our hospital. There is a need for an online system for requesting follow-up appointments in our hospital. This is vital given the 24 hour environment that we work in with many patients being discharged out of normal working hours. This is currently being discussed with management and we hope that the introduction will be imminent to improve the future safety of all patients.
\end{abstract}

\section{Problem}

During the first few weeks on a busy Respiratory/General Medical ward at Ashford and St Peter's NHS Foundation Trust, Chertsey, UK, multiple telephone calls were received both from patients and General Practitioners. They were enquiring as to when investigations or appointments (interventions) were going to happen for recent discharges. It quickly became apparent that despite interventions being listed on the discharge summary they were not all requested or booked. This could lead to missed investigations, undiagnosed illness, investigations not being followed-up or lack of appropriate specialist input for chronic diseases. Ultimately this may lead to poor patient care and impact on patient safety.

There were also incidents on the surgical assessment unit (SAU) when there was a period of time with no ward clerk. This led to no follow-up appointments being booked for any patient discharged from SAU for approximately six weeks. The problem was finally acknowledged and seniors were informed. It was then agreed amongst the doctors that the junior doctors would liaise directly with secretaries to arrange appointments but it quickly became apparent that there were problems outside of normal working hours when secretaries were unavailable. One example is as follows: a patient was discharged after being admitted with abdominal pain thought to be due to gallstones. She was supposed be seen in outpatients to get the results of her investigations but did not, and subsequently was not listed for a cholecystectomy. She presented again to the Accident \& Emergency department with pain and was admitted to the ward. A follow-up appointment would have prevented this repeat admission and would have alleviated the pain caused to this patient. This highlighted that when clerical staff are away, or there are vacant posts, there is a need for an alternative system to enable follow-up appointments to be requested and booked. Junior doctors are always present and, as those responsible for producing discharge summaries, are well placed to request such appointments.

\section{Background}

During induction to the hospital for incoming foundation doctors, there was no formal guidance about arranging follow-up interventions. It was relied upon the senior doctors more familiar with the hospital systems to pass on information to the new doctors. It was generally accepted on the medical ward that the ward clerk would arrange follow-up appointments and doctors would organise investigations. It was well known that the task of compiling the discharge summaries fell to the most junior members of the team (often without appropriate guidance from seniors). For example, all those admitted with an infective exacerbation of COPD should be referred to the respiratory care team on discharge and be seen in the respiratory clinic within four weeks of discharge with a chest $x$ ray on arrival. As junior doctors we would rely on senior colleagues to inform us of protocols such as this one. Decisions about follow up were made at the time the patient was being discharged and when the junior doctor was writing the discharge summary, so this would be the ideal time to put in follow up arrangements. However guidance was not always available and as junior doctors we proposed that more specific guidance was needed in this essential area to prevent poor follow-up.

\section{Baseline}

Our online discharge summary programme was used to identify the last 20 patients discharged from the ward in October. Notes were screened retrospectively for all investigations or appointments that were desired upon discharge. These were recorded and compared with the discharge summary produced for that admission. Over the next 3-4 months the computer programmes; WinPath (a Laboratory Information Management System), Centricity (a radiology software 
programme) and PAS (a Patient Administration System), were used to find out if each intervention on the discharge summary had occurred, when, and how this compared to the desired time period on the discharge summary.

\section{Design}

The follow-up prompt sheet was introduced into the notes of all patients admitted onto the ward over a three week period after which a further 20 records from November were reviewed in the same way as detailed above. The follow-up prompt was a simple A4 sheet with space for patient details at the top and a table with the following headings; Speciality? Intervention? Within what time period?

The follow-up prompt sheet was placed at the back of the current admission medical notes and doctors were informed. Any visiting specialties were also introduced to the prompt and asked to utilise it as necessary. The sheet was filled-in throughout the admission and then used upon discharge to ensure that all interventions for each patient were requested/arranged in an appropriate time frame.

\section{Strategy}

Comparisons between the two sets of patients were made looking particularly at the number of interventions that made it to the discharge summary, the number that actually occurred and whether they happened in the desired time period stated on the discharge summary.

\section{Post-Measurement}

The introduction of a follow-up prompt increased the number of interventions that were recorded on the discharge summary from $93.8 \%$ to $100 \%$ as derived from the comparison of the medical notes with the discharge summaries produced for that admission. The interventions on the discharge summary were divided into primary and secondary care. Of the secondary care interventions, successful follow-ups increased from $73 \%$ to $79 \%$. Patients who were readmitted within the time frame specified for interventions to be performed within were excluded as this meant they were unable to attend appointments or the required interventions had to be expedited due to a change in clinical need. Those who did not attend appointments or refused to attend follow-ups were also excluded. On average $20 \%$ of follow-up arrangements on discharge summaries were requests to GPs recorded on the discharge summary in the section for GP action. These ranged from checking blood tests to arranging further investigations. $75 \%$ of blood tests were initially followed up appropriately and this increased to $100 \%$ with the introduction of the follow-up prompt. Mean time to follow-up was 26 days prior to the prompt (range 72) and 24 days after (range 58). The audit extended for more than three months after patients were discharged, a time frame within which all interventions listed should have occurred. The computer systems could also show any future appointments and this was also taken into consideration. Despite better follow up rates overall the percentage of patients that were followed-up within the expected time frame (as specified on the discharge summary) was $63.2 \%$ prior to the prompt but only $37.5 \%$ after. This may have been due to an increase in the number of interventions requested or unrealistic expectations of the current system's ability to meet demand. It should be noted that this was a small scale audit so this may be an anomaly.

It is clear that not all patients require follow-up after an inpatient stay, $15 \%$ of patients did not have any follow-up listed prior to the follow up prompt compared with just $5 \%$ after. This may reflect that prior to the follow-up prompt some patients were discharged without any interventions arranged despite it being indicated clinically which could have lead to poor patient care.

See supplementary file: Improving Patient Follow Up data.docx

\section{Lessons and Limitations}

Studies that use patient notes are time consuming, requiring organisation and patience. It was sometimes difficult to track notes down; they could be on a different site or in another department within the hospital. This project enforced the well-known need for accurate, legible documentation, as this was paramount for the data collection. The authors now have a better knowledge of the allocation of responsibility when it comes to arranging follow-up and realise just how important it is to have a good handover when starting a new job or specialty. Junior doctors need guidance about discharge plans from experienced colleagues and support from seniors when it comes to trying to make a long-lasting change to a current system. This support is vital when trying to bring about change.

\section{Conclusion}

Timely and appropriate patient follow-up is essential for the future care and safety of our patients following discharge. We have a duty, as doctors, to make sure that these arrangements are made and it is clear from this study that a change is needed in our current system. After discussions with colleagues, there was a consensus that an online system would be most appropriate and given the times at which patients are discharged and that doctors work, there is a need for access to appointments 24 -hours a day.

\section{References}

None. 